\title{
Medical Treatment for Acromegaly does not Increase the Risk of Central Adrenal Insufficiency: A Long-Term Follow-Up Study
}

Authors

Affiliations
F. Ceccato ${ }^{1}$, L. Lizzul ${ }^{1}$, M. Zilio ${ }^{1}$, M. Barbot ${ }^{1}$, L. Denaro ${ }^{2}$, E. Emanuelli ${ }^{3}$, L. Alessio ${ }^{4}$, G. Rolma ${ }^{5}$, R. Manara ${ }^{6}$, A. Saller ${ }^{7}$, M. Boscaro ${ }^{1}$, C. Scaroni ${ }^{1}$

Affiliation addresses are listed at the end of the article

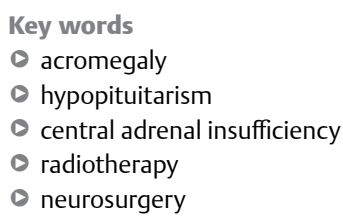

received $\quad 08.12 .2015$

accepted 18.02.2016

\section{Bibliography}

DOI http://dx.doi.org/

10.1055/s-0042-103933

Published online:

May 31, 2016

Horm Metab Res 2016;

48: 514-519

(c) Georg Thieme Verlag KG

Stuttgart · New York

ISSN 0018-5043

\section{Correspondence}

\section{F. Ceccato, MD}

Department of Medicine DIMED

Endocrinology Unit

Via Ospedale Civile 105

35128 Padova

Italy

Tel.: + 39/49/8212559

Fax: + 39/49/657 391

ceccato.filippo@gmail.com

\section{Abstract \\ $\nabla$}

Central adrenal insufficiency (CAI) in acromegaly may be related to pituitary adenoma or induced by various medical treatments, transsphenoidal neurosurgery (TNS) or radiotherapy (RT), alone or combined. We assessed the role of all available treatments for acromegaly in inducing CAI. We retrospectively studied 97 patients. CAI was diagnosed if morning serum cortisol was $<138 \mathrm{nmol} / \mathrm{l}$, or if its response was inadequate in the low-dose short synacthen test. Seventy-four subjects underwent TNS (and 17 of whom also underwent RT), and 23 were on primary medical therapy: overall we diagnosed 21 cases of CAI. Duration of acromegaly, invasion of cavernous sinus, disease control, and type of medical treatment were much the same for patients with and without CAI, which was identified in $18 \%$ of

\section{Introduction}

Acromegaly is caused by a GH-secreting pituitary adenoma. Currently available therapeutic options for its treatment include transsphenoidal neurosurgery (TNS), medication with somatostatin analogues (SSA), dopamine agonists (DA) such as cabergoline, or pegvisomant, and radiotherapy (RT) [1]. Hypopituitarism, severe enough to require replacement therapy [2], can occur both before and after treatment for acromegaly as a result of the adenoma damaging the pituitary gland, or secondary to the effects of the different treatments on pituitary cells $[1,3,4]$. The impact of central adrenal insufficiency (CAI) in acromegaly is still not entirely clear because some authors reported its occurrence only in patients who had undergone TNS, with or without medical treatment, and others excluded irradiated patients from their analyses [3-7]. Yedinak et al. recently wrote that the prevalence of CAI after surgery was lower in patients with acromegaly than in patients (10/57) after one TNS, and in 53\% (9/17) after RT $(\mathrm{p}=0.01)$; repeat surgery increased the risk of CAI $(p=0.02)$. The risk of CAI onset during the follow-up was lower among patients treated with TNS or medical therapy than after RT $(p=0.035)$. Medical treatment did not raise the risk of CAI, whereas a 5- and 4-fold higher risk of CAI was associated with repeat TNS and RT, respectively. Basal or stimulated cortisol levels were similar among acromegalic patients without CAI and matched controls with nonsecreting pituitary lesions. A significant proportion of patients with acromegaly developed CAI over time. While primary or secondary medical treatment did not contribute to the risk of CAI, repeat TNS and RT correlated with pituitary-adrenal axis impairment.

Supporting Information for this article is available online at http://www.thieme-connect.de/products

cases of nonsecreting adenoma: the ACTH-adrenal axis recovered better in acromegalic patients, despite their need for medical therapy to control GH and IGF-1 excess [6]. A higher mortality rate in irradiated acromegalic patients and in those who developed CAI has also been reported [7]. There is still a shortage of data on other aspects, such as the effect of long-term primary medical treatment on hypothalamic-pituitary-adrenal (HPA) axis function.

Our aims were to study a large cohort of acromegalic patients in terms of: (a) the prevalence of CAI; and (b) the influence of different treatments (TNS, medical therapy or RT) on the onset of CAI.

\section{Patients and Methods \\ $\nabla$}

Patients

This retrospective cross-sectional study involved 97 acromegalic patients with normal HPA axis 
function at diagnosis of acromegaly, 38 males and 59 females, with a mean age of $60 \pm 13$ years (range 31-92). Their mean age at the time of their diagnosis was $46 \pm 13$ years (range 24-78) and they had a mean follow-up (as at December 2014) of $13 \pm 10$ years. We considered only those patients with a complete followup and who regularly come to our attention in Pituitary Medical Clinics, we discarded those cases with incomplete follow-up.

Acromegaly was managed according to current international criteria $[1,8]$. The diagnosis was based on: clinical characteristics; inadequate $\mathrm{GH}$ suppression $(<0.4 \mu \mathrm{g} / \mathrm{l})$ after an oral glucose tolerance test (OGTT); and high IGF-1 levels for gender and age. Disease activity was judged on the last available visit (until December 2014). We considered the disease as "active" when randomly-measured serum GH was $\geq 1 \mu \mathrm{g} / \mathrm{l}$ or IGF- 1 levels were below the upper limit of normality in patients with clinical symptoms of active acromegaly, and the GH nadir after OGTT was $\geq 0.4 \mu \mathrm{g} / \mathrm{l}$. Acromegaly was judged to be "controlled" when randomly-measured GH levels were $<1 \mu \mathrm{g} / \mathrm{l}$ and IGF-1 values were within normal range [8]. Remission in patients treated with pegvisomant was judged from normal IGF1 levels. Magnetic resonance (MR) 1.5T scanning was done with a standard quadrature head coil (Achieva; Philips Medical Systems, Best, Netherlands) before and after administering gadolinium contrast. Adenoma was classified by maximal diameter as micro-adenoma $(<10 \mathrm{~mm})$ or macro-adenoma $(\geq 10 \mathrm{~mm})$, and cavernous sinus invasion was assumed from MRI evidence (as explained elsewhere, [9]), or diagnosed during surgery (using the transsphenoidal approach, performed at the local neurosurgery unit). As for medical treatments, acromegalic patients were given SSA (octreotide LAR or lanreotide autogel), DA (cabergoline), or pegvisomant (that is only allowed in Italy for patients who have undergone TNS).

The study was conducted in accordance with the Declaration of Helsinki; our local ethics committee approved the protocol; and all patients gave their informed consent.

\section{Assessment of pituitary deficiencies}

Hormones were measured in the same laboratory at the University Hospital of Padova. From 1982 to 2004 serum cortisol was measured by RIA (DIA-Sorin Diagnostics, Saluggia, Italy) with intra- and interassay coefficient of variation (CV) values of 5.4 and $9.6 \%$, respectively [10]; since 2004 serum cortisol assays are performed with a commercial chemiluminescence immunoassay with declared intra-/interassay CV less than $7 \%$ and less than $9 \%$, respectively [11]. GH was measured with immunoradiometric assay (IRMA) and INSIK-5 (Sorin, Saluggia, Italy) with detection limits of at least $0.2 \mu \mathrm{g} / \mathrm{l}$; IGF-1 was measured with radioimmunoassay (Nichols Institute, San Clemente, CA, USA) for with a detection limit at least of $1.5 \mu \mathrm{g} / 1$ [12] until 2003, then serum GH assays was performed with chemiluminescence by GH IMMUNOLITE 2000 (Siemens, the detection limit was $0.05 \mu \mathrm{g} /$, intra- and interassay variation coefficients of 4.0 and $6.5 \%$, respectively) and serum IGF-1 was measured by chemiluminescence with reagents supplied by DiaSorin Liaison (detection limit was $0.6 \mu \mathrm{g} / \mathrm{l}$, with the intra- and interassay variation coefficients of 5.6 and $7.7 \%$, respectively) [13].

We assessed in all 97 patients basal morning (07:00-09:00 AM) serum cortisol at diagnosis, and then every 12-18 months during the follow-up, or whenever cortisol deficiency was clinically suspected (serum cortisol was checked back the next morning if too high or too low, considering pulsatile secretion). CAI was diagnosed if $\mathrm{F} 0<138 \mathrm{nmol} / \mathrm{l}$, or if cortisol response to low-dose short synacthen test (LDSST) was inadequate (F30 $<440 \mathrm{nmol} / \mathrm{l}$ [14], or F30 $440-600 \mathrm{nmol} / \mathrm{l}$ coinciding with clinical signs or symptoms of cortisol deficiency).

Since 2009, we have systematically performed LDSST in all patients without CAI, considering both basal (F0) and $30 \mathrm{~min}$ (F30) cortisol levels after $1 \mu \mathrm{g}$ ACTH injection. The number of patients with such characteristics was 52, in 30 of whom, thanks to patient's compliance with the strict follow-up, we prospectively studied HPA axis with a standardized protocol based on LDSST at the baseline, 3-6 months after neurosurgery (or after primary medical treatment), and yearly thereafter. The test involved injecting $1 \mu \mathrm{g}$ of synacthen into an antecubital vein between 08:00 and 09:00 AM, taking blood samples for cortisol assay before and again $30 \mathrm{~min}$ after the injection. The concentration of $1 \mu \mathrm{g} / \mathrm{ml}$ was obtained by diluting synacthen (available in Italy in $250 \mu \mathrm{g} / \mathrm{ml}$ vials) in $249 \mathrm{ml}$ of sterile saline solution.

As a control group, we recruited 37 patients with nonfunctioning pituitary lesions (adenoma $n=32$; Rathke cleft cyst $n=5$ ) and no CAI, who were matched with the acromegalic patients for age, sex, and treatment history (TNS, RT, or no treatment).

Pituitary deficiencies were diagnosed in all acromegalic patients, as described elsewhere [15]; briefly, GH deficiency was confirmed by a GHRH + arginine stimulation test with cut-offs based on BMI. The diagnosis of other hormone deficiencies (central hypothyroidism or hypogonadism) was based on low pituitary hormone levels with low serum fT4 or gonadal steroid hormones.

\section{Statistical analysis}

We calculated proportions and rates for categorical variables, and means and standard deviations, or medians and inter-quartile ranges (IQR) for parametric or nonparametric variables, respectively. Groups were compared with the chi-square test for categorical variables (or Fisher's exact test when the cell count was $<5$ ), and Student's t-test or the Mann-Whitney test for quantitative variables, as appropriate. HPA axis integrity during the follow-up was established from the Kaplan-Meier curves and compared with the log rank test; a binomial logistic regression analysis was used as a multivariate test to calculate the hazard ratio (HR). The SPSS 17 software package (SPSS, Inc., Chicago, IL, USA) was used for all analyses. The significance level was set at $\mathrm{p}<0.05$ for all tests.

\section{Results \\ $\nabla$ \\ CAl in acromegalic patients}

The patients' clinical characteristics are summarized in $\bullet$ Table 1. The overall prevalence of CAI in our cohort was 22\% (21 out of 97 patients), and it was found unrelated to age, gender, age at diagnosis, duration of follow-up or cavernous sinus invasion, although CAI patients had larger adenomas. Two of our patients presented CAI at acromegaly diagnosis, and so were excluded from further analyses.

Considering treatments, in our cohort 23 acromegalic patients were treated with primary medical therapy ( 21 with SSA, 2 with DA; median 8 years; IQR 3.5-12). The other 74 patients underwent TNS (51 once), and among surgical patients 24 assumed medical treatment before TNS (for 3-6 months). Surgical failure was observed in $39 \%$ of patients, who then assumed medical therapy (SSA, DA or pegvisomant, alone or combined), 6 repeated TNS and 17 were irradiated (treatment strategies are summarized in $\odot$ Fig. 1 ). 
Table 1 Clinical characteristics of acromegalic patients with and without central adrenal insufficiency (CAI).

\begin{tabular}{llll} 
Characteristic & $\begin{array}{l}\text { With CAI } \\
(\mathbf{n = 2 1 )}\end{array}$ & $\begin{array}{l}\text { Without CAI } \\
\text { (n=76) }\end{array}$ & p \\
\hline Age at acromegaly diagnosis (years) & $41(33-51)$ & $49(38-57)$ & 0.107 \\
\hline Age at time of study (years) & $64(46-67)$ & $60(51-70)$ & 0.673 \\
\hline Follow-up for acromegaly (months) & $192(84-264)$ & $108(60-204)$ & 0.122 \\
\hline Female (\%) & $14 / 21(67)$ & $48 / 81(59)$ & 0.536 \\
\hline Adenoma max diameter (mm) & $21(15-23)$ & $15(10-16)$ & $\mathbf{0 . 0 1 0}$ \\
\hline Pituitary macroadenoma (\%) & $11 / 13(85)$ & $44 / 65(68)$ & 0.324 \\
\hline Cavernous sinus invasion (\%) & $19 / 21(90)$ & $57 / 81(70)$ & 0.225 \\
\hline Pituitary TNS (\%) & $19 / 21(91)$ & $55 / 81(68)$ & 0.053 \\
\hline Pituitary TNS (without RT) & $10 / 12(83)$ & $47 / 72(65)$ & 0.322 \\
\hline SSA (\%) & $14 / 21(67)$ & $50 / 81(62)$ & 0.802 \\
\hline SSA as primary treatment & $2 / 2(100)$ & $19 / 21(90)$ & 0.397 \\
\hline SSA after TNS & $12 / 19(63)$ & $31 / 55(56)$ & 0.605 \\
\hline Dopamine agonist (\%) & $4 / 21(19)$ & $17 / 81(21)$ & 1.000 \\
Pegvisomant & $5 / 21(26)$ & $15 / 81(27)$ & 0.935 \\
\hline Pituitary RT (\%) & $9 / 21(43)$ & $9 / 81(11)$ & $\mathbf{0 . 0 0 1}$
\end{tabular}

Data are shown as medians (IQR) or percentages

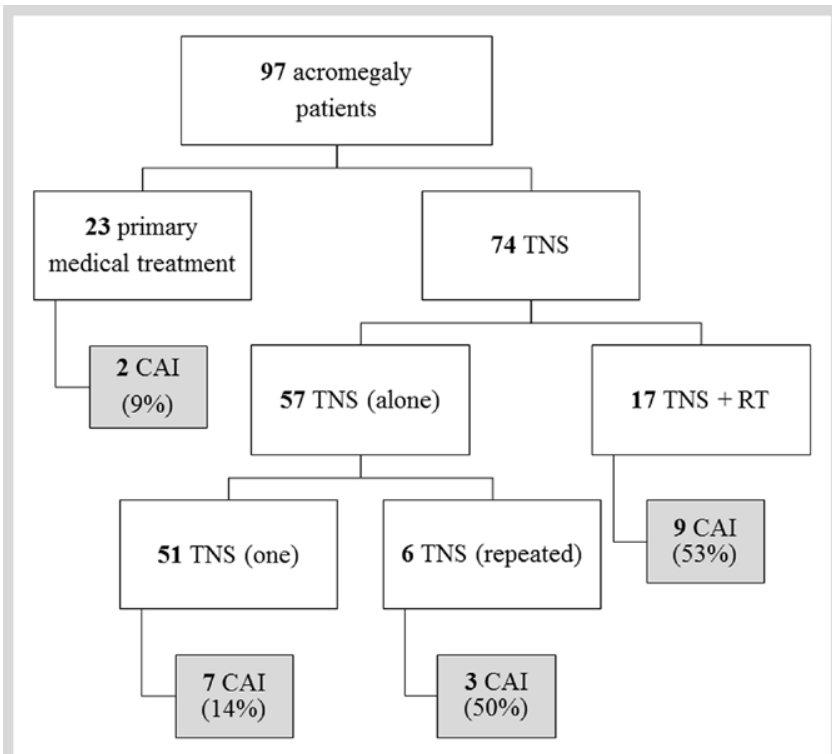

Fig. 1 Study design and CAl distribution among the various treatments.

As regards medication, the use of SSA, DA, or pegvisomant was similar for patients with and without CAI, for both primary and post-TNS therapy: the CAI onset rate was lower after primary medical treatment than after repeat TNS $(p=0.002)$ or RT $(p=0.003)$. Patients who underwent TNS without any subsequent RT revealed no HPA axis damage $(p=0.3)$, whereas $50 \%$ of patients who repeated TNS developed CAI ( $p=0.015$ vs. patients who had TNS only once).

In our cohort, 9 out of 17 of irradiated patients (after TNS) developed CAI: after conventional RT in 8/13 cases, and after radiosurgery in $1 / 4(\mathrm{p}=0.2)$. Pituitary irradiation was associated with a higher risk of developing CAI than TNS plus medical therapy, as shown in $\odot$ Fig. 2 (Mantel-Cox log rank $\mathrm{p}=0.035$ for TNS + RT vs. TNS, and $\mathrm{p}=0.034$ for TNS + RT vs. medical treatment). In a binomial logistic regression analysis performed to calculate the HR of each treatment inducing CAI, RT, and repeat TNS carried the highest risk ( $\odot$ Table 2 ), whereas primary or secondary medical treatment were unrelated to the onset of CAI. Among the 17

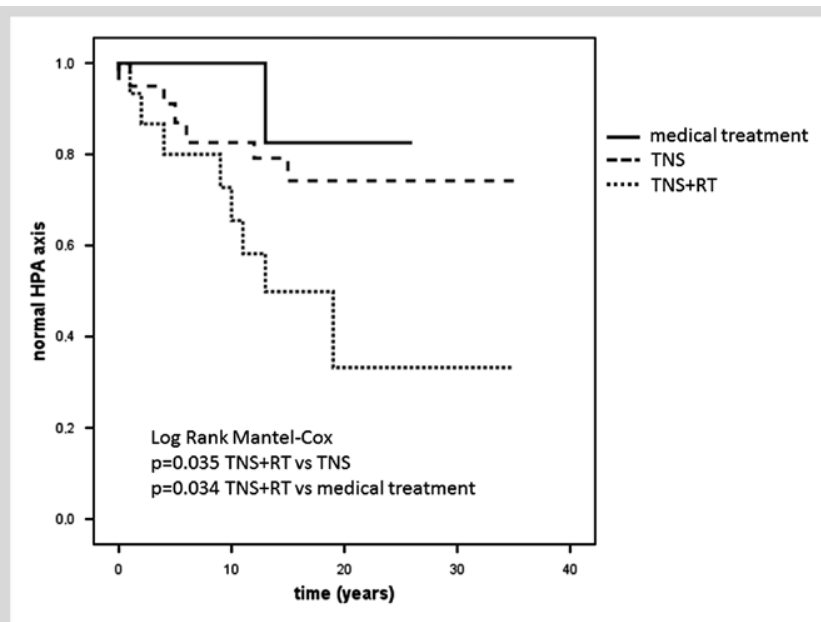

Fig. 2 Life-table analysis indicating probabilities of initially normal HPA axis remaining normal in our cohort by type of treatment.

Table 2 Binomial logistic regression analyses to calculate the role of several variables in inducing CAI.

\begin{tabular}{|lll|} 
& $\mathbf{P}$ & HR (95\% Cl) \\
\hline Gender & 0.404 & $1.71(0.48-6.05)$ \\
Cavernous sinus invasion & 0.452 & $2.41(0.24-23.93)$ \\
\hline Medical treatment & 0.368 & $1.81(0.49-6.59)$ \\
TNS & 1.00 & $0.00(0.00-0.02)$ \\
\hline Repeat TNS & $\mathbf{0 . 0 3 7}$ & $\mathbf{5 . 8 4}(1.11-30.62)$ \\
\hline Radiotherapy & $\mathbf{0 . 0 4 4}$ & $\mathbf{4 . 0 1}(1.04-15.46)$ \\
\hline
\end{tabular}

HR: Hazard ratio; Cl: Confidence interval for HR

patients irradiated after TNS, the CAI rate was statistically similar among the 13 patients who had only one TNS ( 6 developed CAI, $46 \%$ ) and among the 4 subjects who had repeat TNS before receiving $\mathrm{RT}$ ( 3 developed $\mathrm{CAI}, 75 \%, \mathrm{p}=0.312$ ).

The rate of other pituitary deficiencies was higher for the acromegalic patients with CAI than for those judged to have a normal HPA axis $(p=0.001)$, especially among the irradiated patients $(\mathrm{p}=0.001)$.

\section{Acromegaly control and CAI}

Overall, acromegaly was controlled in $80 \%$ of our patients $(>90 \%$ after $\geq 5$ years of follow-up), irrespective of adenoma size (73\% of macro-adenomas and $90 \%$ of micro-adenomas, $\mathrm{p}=0.778$ ), use of TNS ( $81 \%$ with vs. $75 \%$ without TNS, $p=0.585$ ), medical treatment (75\% with vs. $87 \%$ without medical treatment, $\mathrm{p}=0.207$ ), or RT ( $94 \%$ with vs. $77 \%$ without RT, $\mathrm{p}=0.082$ ). The acromegaly control rate was similar for patients given RT after TNS and those who underwent surgery alone ( $90 \mathrm{vs.} 78 \%, \mathrm{p}=0.118$ ). Acromegaly control was also similar in patients with and without CAI (86 vs. $78 \%, p=0.423$ ), also taking any use of TNS or RT into account.

\section{LDSST results in patients and controls}

The LDSST was performed at least once in 52 acromegalic patients with a normal HPA axis (13 on primary medical therapy, 33 after TNS, and 6 after TNS + RT), who were matched with 37 patients with non-GH secreting pituitary lesions and a similar treatment history (11 had neither TNS nor RT, 20 underwent TNS, and 6 had TNS+RT). The LDSST revealed CAI in 6 patients with normal basal cortisol levels (as summarized in supplementary Table 1S). 

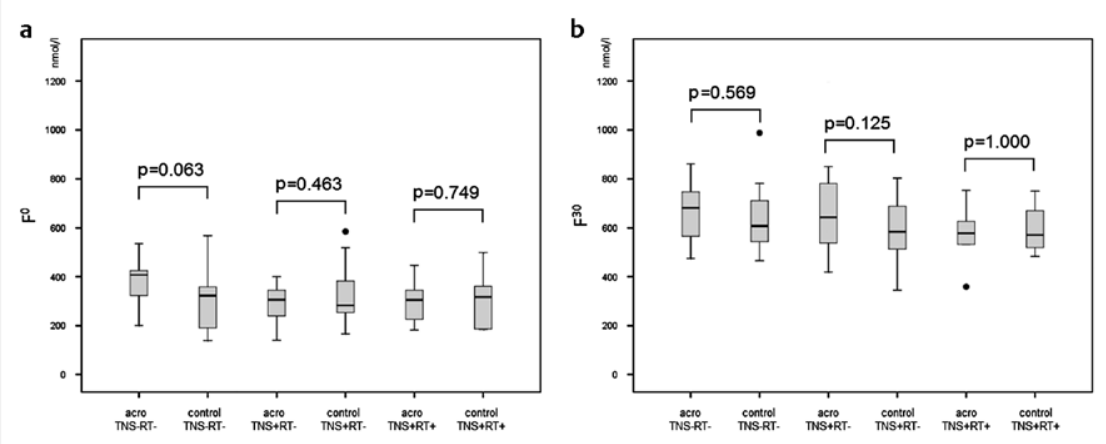

Fig. 3 Cortisol levels at F0 (panel a) and F30 after LDSST (panel b) among acromegalic patients (with a normal HPA axis) and controls matched for treatment history.

As shown in $\bullet$ Fig. 3, we found no differences in LDSST results between acromegalic patients without CAI and controls (median F0 313 vs. $387 \mathrm{nmol} / \mathrm{l}$, and median F30 631 vs. $588 \mathrm{nmol} / \mathrm{l}$, respectively; $\mathrm{p}=0.612$ and $\mathrm{p}=0.163$ ). We also found no differences in the cortisol levels found at F0 and F30 between the first and the last available LDSST ( 30 subjects, with a median 3 years elapsing between the 2 tests).

\section{Discussion}

$\nabla$

The onset of CAI in acromegaly could be due to damage induced either by the pituitary adenoma or by its treatment, be it medical therapy, TNS, or RT [1,3]. Its prevalence varies widely, depending on the different types of treatment administered and on the inclusion criteria considered in different studies $[3,5,6]$. Some facets of acromegaly are still unclear, such as the effect of long-term medical treatment on HPA axis function. Hence, our study on the prevalence of CAI and the role of various treatments in a large cohort of acromegalic patients referred to a single tertiary center.

We describe a large series of consecutive, unselected patients who could access all available treatments, and who had a lengthy median follow-up (mean 13 years). We found a high overall prevalence of CAI (22\%), similar to the proportions reported by Ronchi et al. in 36 cured acromegalic patients ( $32 \%$ of them with ACTH impairments over a median follow-up of 6 years, considering RT as an exclusion criterion [3]), and by Sherlock et al. (36\% of CAI in acromegaly [7]). On the other hand, Burgers et al. studied 91 patients who were cured after one surgical procedure (none of whom were given medical therapy or RT) and found that only $9 \%$ had developed CAI by the end of the first year after surgery, and $12 \%$ during an 8-year follow-up [5]; and Mercado et al. likewise reported a $9 \%$ rate of CAI [16]. Yedinak et al. recently published a systematic 1 -year study based on LDSST in 50 acromegalic patients after TNS (excluding those given RT): they described a $20 \%$ prevalence of CAI, despite medical treatment [6]. We did not see a rise in the rate of CAI among patients given medical treatment, not even after a long follow-up, and considering all available drugs. We also studied the effect of medical therapy both as primary treatment (when TNS was contraindicated [17]) and after TNS proved unsuccessful or while awaiting the effects of RT. Two patients developed CAI after primary medical therapy with SSA: although there have been reports of somatostatin inhibiting ACTH secretion [18], SSA treatment did not affect HPA axis integrity in our cohort. In the present study we considered octreotide or lanreotide, but not pasireotide (the latest SSA to become available for acromegaly), so it will be interesting in future to examine the effects of this last drug on corticotroph cells, given its greater affinity for SSA receptor 5, and consequent potential effect on ACTH secretion [19]. To our knowledge, there is currently no data available on the risk of cabergoline (and other DAs), or pegvisomant inducing CAI in acromegaly, nor any information on their role after unsuccessful TNS [20-22].

Our acromegalic patients who underwent TNS only once did not have a higher rate of CAI than those in medical treatment, providing they received no RT. In our cohort the presence of cavernous sinus invasion, which could be taken as a sign of large and aggressive tumors (usual candidates for repeated surgery or RT), was not associated with CAI, probably because we choose medical treatment to control both adenoma size and secretion. On the other hand, resurgery was clearly associated with the onset of CAI, however, with a 5-fold higher risk of its occurrence after multiple surgical procedures (50\% of such patients developed ACTH deficiency). Of course, patients undergoing repeated TNS or given RT had clinical features of a more "aggressive" GHsecreting pituitary adenoma. Ten of our patients had repeated TNS, and 4 of them were also irradiated. In this subgroup, acromegaly was judged to be under control in 6 of the 10 patients as at their latest follow-up, 3 were also irradiated (and developed CAI), 8 had a macroadenoma with cavernous sinus invasion at diagnosis, and one carried an aryl hydrocarbon receptor-interacting protein (AIP) mutation, and loss of AIP function or expression may contribute to pharmacological resistance [23].

As expected, pituitary RT coincided with a 4-fold higher risk of CAI [7]. This risk effect increased over time, the delay probably being related to a progressive fibrotic degeneration and/or vascular remodeling after irradiation, which can result in of HPA axis derangement. The Kaplan-Meier curve for HPA axis integrity showed that RT correlated with CAI more closely than TNS or medical therapy. The onset of CAI in irradiated patients was unrelated to the number of TNS performed before RT, whether acromegaly was controlled or not. Judging from our results, we would suggest medical therapy (wherever possible) after unsuccessful surgery, rather than RT, with a view to preserving the HPA axis, bearing in mind that both RT and ACTH deficiency are strongly related with a higher mortality rate in acromegaly [7]. Basal and stimulated cortisol levels after LDSST were much the same in our acromegalic patients without CAI (52 cases) and controls matched for treatment history, in terms of TNS or RT $(n=37)$, meaning that the natural history of the HPA axis in acromegaly is comparable with the situation seen in other pituitary diseases. LDSST probably is able to establish CAI diagnosis especially in patients after RT, when we observe a gradual decline in ACTH function rather than a sudden onset of CAI (as after TNS). 
We saw no differences in basal and stimulated cortisol levels between patients' first and last available LDSST findings, but we collected data over a median 3 years, which is probably not long enough for any HPA axis impairment to come to light. It has now been well established that diagnosing CAI is a critical issue due to the lack of reliable tests, and difficulties in their interpretation. In our series, we have recently performed LDSST systematically, and this has led to CAI being diagnosed more often because even mild forms are identified, when the signs and symptoms are still vague. The insulin tolerance test is considered the gold standard for diagnosing CAI, but is not used routinely (in our cohort we performed a reduced number of insulin test as well). The LDSST is quick, easy and safe, assuring a good diagnostic accuracy when reference thresholds are adopted $[14,24]$. Patients with intermediate values may naturally warrant further testing, or a careful search for any clinical signs of CAI.

Our work has some limitations. First, the follow-up was not standardized, since we have used LDSST only in recent years. Other tests were performed in the past (especially high dose ACTH, CRH or insulin test), but only in a small number of subjects, or when the CAI diagnosis was based especially upon morning serum cortisol or clear clinical signs of hypoadrenalism. In addition, we found no differences between conventional RT and radiosurgery, probably because the latter method was introduced quite recently so only a few patients received this treatment and probably they did not have enough time to develop CAI yet. Moreover, some patients were lost in follow-up, and the presented results could be referred only to the survivors who regularly came to be examined in our clinic.

In conclusion, we found that HPA axis function may deteriorate over time - regardless of whether or not acromegaly is under control - especially after second TNS or RT. On the other hand, medical treatment was unrelated to any onset of CAI in our cohort, whether it was used as a primary therapy or after TNS. Measuring basal cortisol is of the utmost importance, but the LDSST can pinpoint additional patients with CAI. The HPA axis should therefore be carefully assessed in all patients at regular intervals, and treatment strategies to achieve acromegaly control should balance hormone excess with the risk of hypopituitarism.

\section{Conflict of Interest}

$\nabla$

None of the authors have any conflicts of interest or financial interests to disclose that might be perceived as influencing the impartiality of the reported research.

\section{Affiliations}

'Department of Medicine DIMED, Endocrinology Unit, University Hospital of Padova, Padova, Italy

Department of Neurosciences DNS, Neurosurgery, University Hospital of Padova, Padova, Italy

Otorhinolaryngology and Otologic Surgery, University Hospital of Padova, Padova, Italy

Neurosurgical Division, University Hospital of Padova, Padova, Italy

5 Neuroradiology Unit, University Hospital of Padova, Padova, Italy

${ }^{6}$ Department of Medicine and Surgery, Neuroradiology, University of Salerno, Salerno, Italy

Department of Medicine DIMED, Internal Medicine, University Hospital of Padova, Padova, Italy

\section{References}

1 Melmed S, Colao A, Barkan A, Molitch M, Grossman AB, Kleinberg D, Clemmons D, Chanson P, Laws E, Schlechte J, Vance ML, Ho K, Giustina A. Guidelines for acromegaly management: an update. J Clin Endocrinol Metab 2009; 94: 1509-1517

2 Rushworth RL, Torpy DJ. Modern Hydrocortisone Replacement Regimens in Adrenal Insufficiency Patients and the Risk of Adrenal Crisis. Horm Metab Res 2015; 47: 637-642

3 Ronchi CL, Ferrante E, Rizzo E, Giavoli C, Verrua E, Bergamaschi S, Lania AG, Beck-Peccoz P, Spada A. Long-term basal and dynamic evaluation of hypothalamic-pituitary-adrenal (HPA) axis in acromegalic patients. Clin Endocrinol (Oxf) 2008; 69: 608-612

4 Katznelson L, Laws ERJr, Melmed S, Molitch ME, Murad MH,UtzA, WassJA. Acromegaly: an Endocrine Society clinical practice guideline. J Clin Endocrinol Metab 2014; 99: 3933-3951

5 Burgers AM, Kokshoorn NE, Pereira AM, Roelfsema F, Smit JW, Biermasz $N R$, Romijn JA. Low incidence of adrenal insufficiency after transsphenoidal surgery in patients with acromegaly: a long-term follow-up study. J Clin Endocrinol Metab 2011; 96: 1163-1170

6 Yedinak C, Hameed N, Gassner M, Brzana J, McCartney S, Fleseriu M. Recovery rate of adrenal function after surgery in patients with acromegaly is higher than in those with non-functioning pituitary tumors: a large single center study. Pituitary 2015; 18: 701-709

7 Sherlock M, Reulen RC, Alonso AA, Ayuk J, Clayton RN, Sheppard MC, Hawkins MM, Bates AS, Stewart PM. ACTH deficiency, higher doses of hydrocortisone replacement, and radiotherapy are independent predictors of mortality in patients with acromegaly. J Clin Endocrinol Metab 2009; 94: 4216-4223

8 Giustina A, Chanson P, Bronstein MD, Klibanski A, Lamberts S, Casanueva FF, Trainer $P$, Ghigo E, Ho K, Melmed S. A consensus on criteria for cure of acromegaly. J Clin Endocrinol Metab 2010; 95: 3141-3148

9 Manara R, Maffei P, Citton V, Rizzati S, Bommarito G, Ermani M, Albano I, Della Puppa A, Carollo C, Pavesi G, Scanarini M, Ceccato F, Sicolo N, Mantero F, Scaroni C, Martini C. Increased rate of intracranial saccular aneurysms in acromegaly: an MR angiography study and review of the literature. J Clin Endocrinol Metab 2011; 96: 1292-300

10 Albiger NM, Occhi G, Mariniello B, Iacobone M, Favia G, Fassina A, Faggian $D$, Mantero F, Scaroni $C$. Food-dependent Cushing's syndrome: from molecular characterization to therapeutical results. Eur J Endocrinol 2007; 157: 771-778

11 Ceccato F, Barbot M, Zilio M, Frigo AC, Albiger N, Camozzi V, Antonelli G, Plebani M, Mantero F, Boscaro M, Scaroni C. Screening Tests for Cushing's Syndrome: Urinary Free Cortisol Role Measured by LC-MS/MS. J Clin Endocrinol Metab 2015; 100: 3856-3861

12 Ceccato F, Occhi G, Albiger NM, Rizzati S, Ferasin S, Trivellin G, Mantero $F$, Scaroni $C$. Adrenal lesions in acromegaly: do metabolic aspects and aryl hydrocarbon receptor interacting protein gene have a role? Evaluation at baseline and after long-term follow-up. J Endocrinol Invest 2011; 34: 353-360

13 Fadini GP, Dassie F, Albiero M, Boscaro E, Albano I, Martini C, de Kreutzenberg SV, Agostini C, Avogaro A, Vettor R, Maffei P. Endothelial progenitor cells are reduced in acromegalic patients and can be restored by treatment with somatostatin analogs. J Clin Endocrinol Metab 2014; 99: 2549-2556

14 Kazlauskaite R, Evans AT, Villabona CV, Abdu TA, Ambrosi B, Atkinson $A B$, Choi CH, Clayton RN, Courtney CH, Gonc EN, Maghnie M, Rose SR, Soule SG, Tordjman K. Corticotropin tests for hypothalamic-pituitaryadrenal insufficiency: a metaanalysis. J Clin Endocrinol Metab 2008; 93: 4245-4253

15 Ceccato F, Albiger N, Reimondo G, Frigo AC, Ferasin S, Occhi G, Mantero $F$, Terzolo M, Scaroni C. Assessment of glucocorticoid therapy with salivary cortisol in secondary adrenal insufficiency. Eur J Endocrinol 2012; 167: 769-776

16 Mercado M, Gonzalez B, Vargas G, Ramirez C, de los Monteros AL, Sosa $E$, Jervis $P$, Roldan P, Mendoza V, López-Félix B, Guinto G. Successful mortality reduction and control of comorbidities in patients with acromegaly followed at a highly specialized multidisciplinary clinic. J Clin Endocrinol Metab 2014; 99: 4438-4446

17 Caron PJ, Bevan JS, Petersenn S, Flanagan D, Tabarin A, Prévost G, Maisonobe $P$, Clermont A, PRIMARYS Investigators. Tumor shrinkage with lanreotide Autogel $120 \mathrm{mg}$ as primary therapy in acromegaly: results of a prospective multicenter clinical trial. J Clin Endocrinol Metab 2014; 99: 1282-1290

18 Luini A, Lewis D, Guild S, Schofield G, Weight F. Somatostatin an inhibitor of ACTH secretion, decreases cytosolic free calcium and voltagedependent calcium current in a pituitary cell line. J Neurosci 1986; 6: 3128-2132 
19 Ceccato F, Scaroni C, Boscaro M. Clinical use of pasireotide for Cushing's disease in adults. Ther Clin Risk Manag 2015; 11: 425-434

20 van der Lely AJ, Biller BM, Brue T, Buchfelder M, Ghigo E, Gomez R, HeyHadavi J, Lundgren F, Rajicic N, Strasburger CJ, Webb SM, KoltowskaHäggström $M$. Long-term safety of pegvisomant in patients with acromegaly: comprehensive review of 1288 subjects in ACROSTUDY. J Clin Endocrinol Metab 2012; 97: 1589-1597

21 Marazuela M, Ramos-Leví A, Sampedro-Núñez M, Bernabeu I. Cabergoline treatment in acromegaly: pros. Endocrine 2014; 46: 215-219

22 Freda PU, Gordon MB, Kelepouris N, Jonsson P, Koltowska-Haggstrom M, van der Lely AJ. Long-term treatment with pegvisomant as monotherapy in patients with acromegaly: experience from acrostudy. Endocr Pract 2015; 21: 264-274
23 Jaffrain-Rea ML, Rotondi S, Turchi A, Occhi G, Barlier A, Peverelli E, Rostomyan L, Defilles C, Angelini M, Oliva MA, Ceccato F, Maiorani O, Daly $A F$, Esposito $V$, Buttarelli $F$, Figarella-Branger D, Giangaspero $F$, Spada A, Scaroni C, Alesse E, Beckers A. Somatostatin analogues increase AIP expression in somatotropinomas, irrespective of Gsp mutations. Endocr Relat Cancer 2013; 20: 753-766

24 Grossman $A B$. The diagnosis and management of central hypoadrenalism. J Clin Endocrinol Metab 2010; 95: 4855-4863 